\title{
Protée
}

\section{Échos aériens dans l'oeuvre de Marie-Claude Bouthillier}

\section{Catherine Mavrikakis}

Volume 35, numéro 1, printemps 2007

Échos et résonances

URI : https://id.erudit.org/iderudit/015888ar

DOI : https://doi.org/10.7202/015888ar

Aller au sommaire du numéro

\section{Éditeur(s)}

Département des arts et lettres - Université du Québec à Chicoutimi

\section{ISSN}

0300-3523 (imprimé)

1708-2307 (numérique)

Découvrir la revue

Citer cet article

Mavrikakis, C. (2007). Échos aériens dans l'oeuvre de Marie-Claude Bouthillier. Protée, 35(1), 48-54. https://doi.org/10.7202/015888ar d'utilisation que vous pouvez consulter en ligne.

https://apropos.erudit.org/fr/usagers/politique-dutilisation/ 

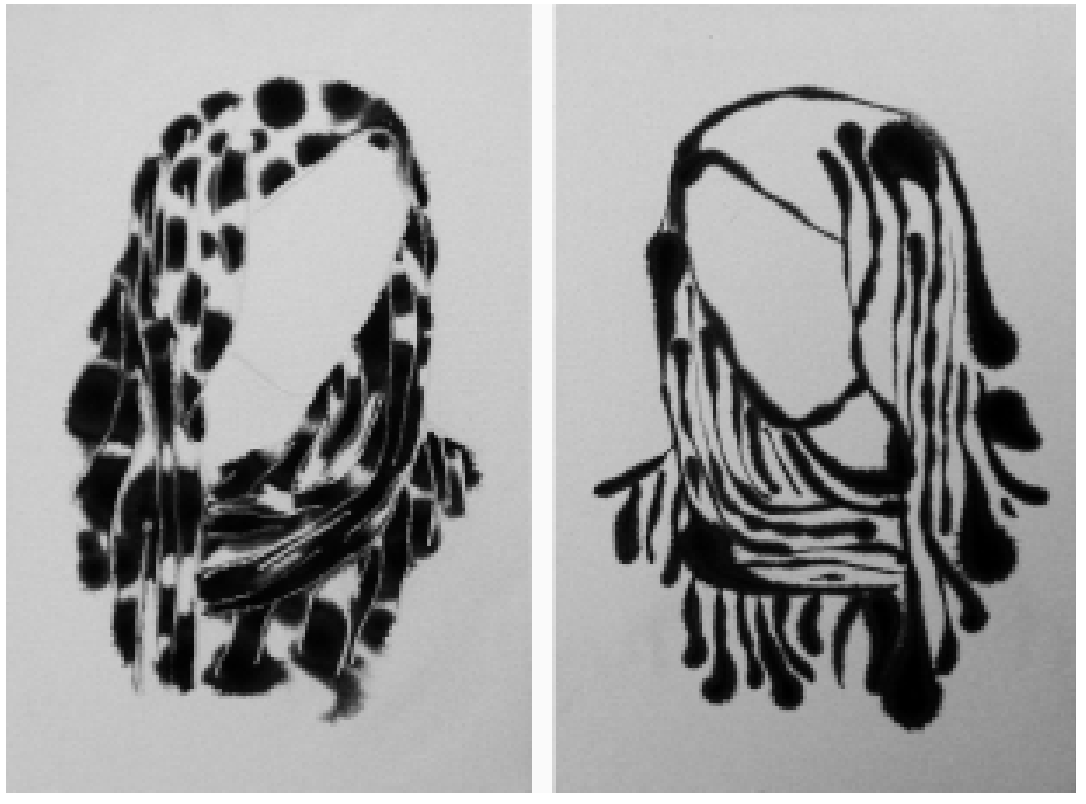

Deux Drapés (diptyque), 2006, 60 × $90 \mathrm{~cm}$ (dispersion de pigment sur toile).

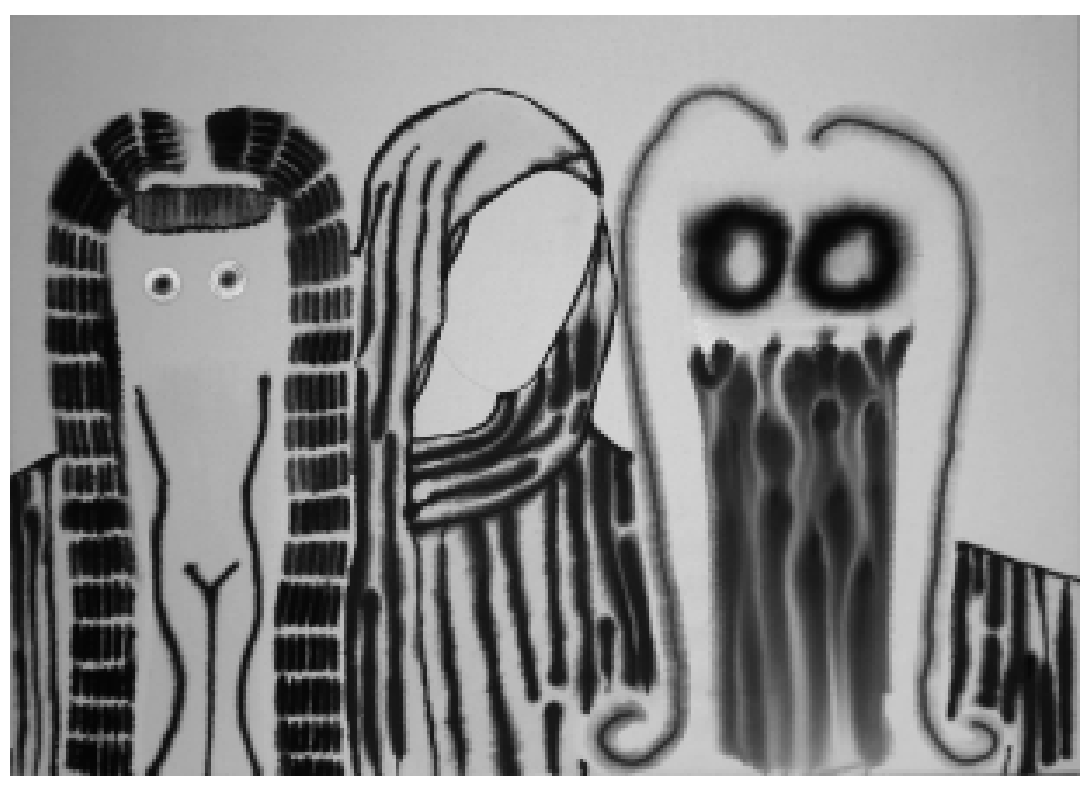

Trinité, 2007, 76 × $107 \mathrm{~cm}$ (acrylique sur toile). 


\title{
ÉCHOS AÉRIENS DANS L'OEUVRE DE MARIE-CLAUDE BOUTHILLIER
}

\author{
Catherine Mavrikakis
}

Il fut un temps où, à partir d'un moi inscrit dans ses initiales, véritable sigle, MarieClaude Bouthillier («mcb») créait des formes. L'image de soi, le reflet patronymique répété jusqu'à sa perte ou encore son engloutissement dans la figure ou l'abstraction qui l'avale, permettait à l'artiste de mettre en place une partie de cache-cache avec le spectateur jouant à trouver et à perdre «mcb» dans ses tableaux et dessins. L'identité était alors ludique et voulait penser les limites du moi et de sa représentation dans sa possible disparition.

Si le mot ainsi devient motif et perd sa valeur singulière pour se transformer en anagramme, en hiéroglyphe puis en simple forme, il se métamorphose encore pour se faire fragment de mouvement, arabesque, volute. La saturation incarnera ici l'apesanteur et la délicatesse. Car il y a quelque chose de la volatilité dans le travail de Marie-Claude Bouthillier. La perte s'inscrit dans la répétition, mais nous avons ici affaire à une perte qui permet au motif réitéré de prendre son envol, de se faire léger. Les dessins semblent être en suspension sur la feuille ou sur la toile, comme s'ils évoluaient dans un espace qui n'arrive pas à les contenir, à leur assigner un contour définitif et à les retenir parmi nous. Quelque chose flotte dans l'œuvre de Bouthillier comme si I'on faisait fi de l'attraction terrestre et que les personnages, tels des ballons ou des saints, étaient susceptibles de s'éclipser, de s'élever vers le ciel, presque désarrimés de leur support. Les dessins s'enflent du blanc qui les entoure ou de la toile qui les porte et se gonflent d'un monde qui les soulève. L'aérien ici ne devient jamais éthéré; il existe par la matérialité et se fonde dans l'emprise parfois maniaque de la répétition.

Autour des dessins de Bouthillier s'installe souvent une bavure de la couleur, une auréole qui confère à la représentation une limite autre, qui permet au tracé de jouer sur son possible déplacé, sur son flou. Dans cette bavure, il faut peut-être voir l'aura des choses, un halo qui permet au dessin d'être à la fois présence, insistance et signe d'une immatérialité, $d^{\prime}$ un effacement progressif. On pourrait très vite dire que cette attention à l'aura des objets et des êtres, conjuguée à cette pratique qui fait écho à l'élévation, à l'assomption virginale ou encore à l'ascension, s'exerce dans un espace religieux, chrétien, savoureusement mobile. Certaines œuvres dont les titres sont Trinité ou encore Saintes-Grenouilles font signe à un religieux travesti, à une anamorphose du divin digne du roman Notre-Dame-des-Fleurs de 
Jean Genet. Mais cette reprise souriante du monde divin chrétien n'est jamais chargée du poids de I'Histoire. Au contraire... II y a du rupestre dans les œuvres de Marie-Claude Bouthillier. L'art ici tient parfois du pétroglyphe dans la mesure où y apparaît un primitif ou un archaïque (et, par là, je rejette tout écho négatif à l'art naïf) qui travaille sur une « visagéité » originelle ou une corporéité schématique. Certaines œuvres s'apparentent aux tracés sur les parois d'une grotte moderne. S'il y a du dédoublement, du redoublement, de la multiplication ou encore de la sérialité dans le travail de Bouthillier, ce sériel doit être considéré comme désordonné. On assiste à une répétition où surgit de l'imprévu déjouant les pièges du mécanisme de reproduction et de la machine à produire du même. L'œuvre fait signe à un dépouillement de l'origine dont la répétition révèle l'élémentaire et l'essentiel. Le paradoxe tient à ce que la répétition ne vient nullement marteler le spectateur, mais, au contraire, lui permet de respirer, d'être surpris, puisqu'il sera toujours étonné par les reprises inusitées, ironiques ou amusées. Il découvrira une bavure, un trait, une forme, une couleur, un point agrandi, amenuisé, transformé, repris dans une autre couleur et il ne saura plus distinguer les originaux des copies dans le pêle-mêle des corps. Bouthillier travaille avec la répétition de gestes, mais, en variant le médium (acrylique, encre, cire...) et le support (papier et toile, mouillés ou non), elle produit différents types de formes et de figures qui se font écho tout en proposant des charades étonnantes. Les œuvres se parlent aussi entre elles, se volant des morceaux, s'empruntant des lignes, des couleurs et se chevauchant ainsi dans les imaginaires. Bouthillier crée de la sorte une impression de déjà-vu taquin tout au long de ses œuvres, sans que celui ou celle qui regarde le tableau ou le dessin soit tout à fait à même de reconnaître avec précision ce qu'il ou elle a vu. Un phénomène de paramnésie provoquée est alors discernable, et Bouthillier joue avec la mémoire sensorielle.

Il est bien sûr ridicule de comprendre l'œuvre d'une artiste, quelle qu'elle soit, à partir d'une notion qu'elle viendrait illustrer. Ici l'écho ne saurait être compris ou mis en scène par une pratique aussi complexe et originale que celle de Bouthillier. Le travail de cette artiste résonne de façon étonnamment familière en s'autorisant à rendre les concepts aériens et ailés.

Le choix des images de Marie-Claude Bouthillier a été fait conjointement avec Nicolas Mavrikakis. 


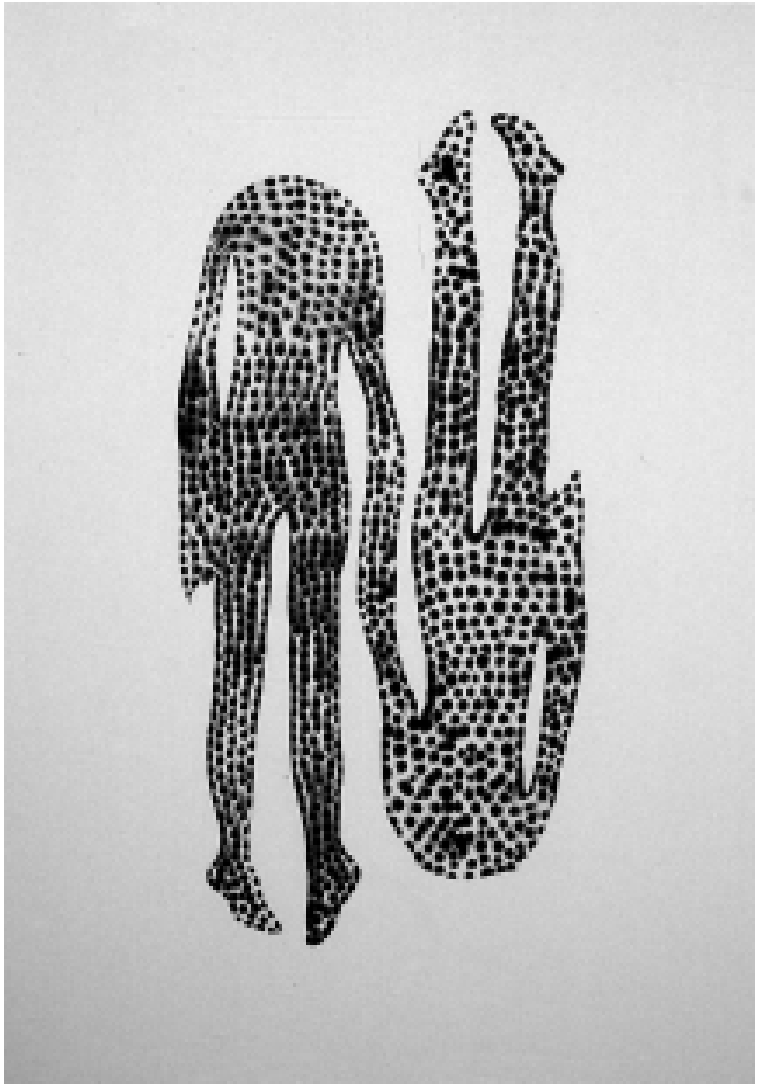

Deux Corps sans tête, 2004,

$320 \times 180 \mathrm{~cm}$ (dispersion de pigment sur toile).

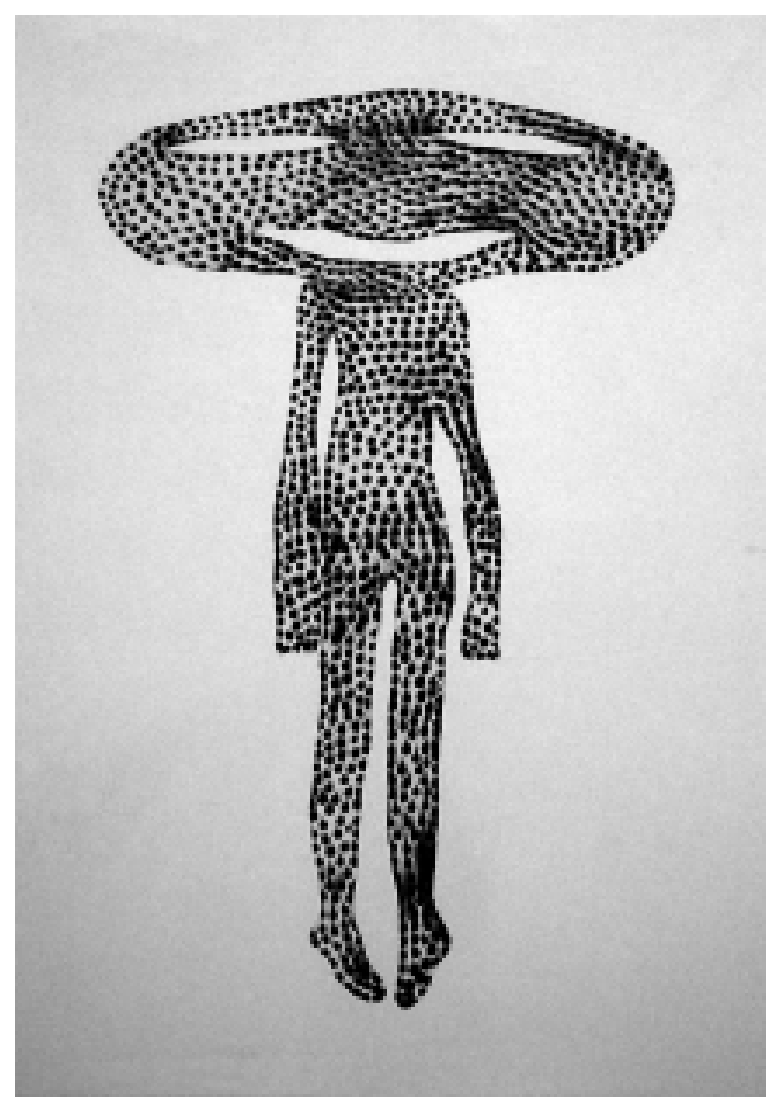

Grand Visage, 2004

$320 \times 180 \mathrm{~cm}$ (dispersion de pigment sur toile). 


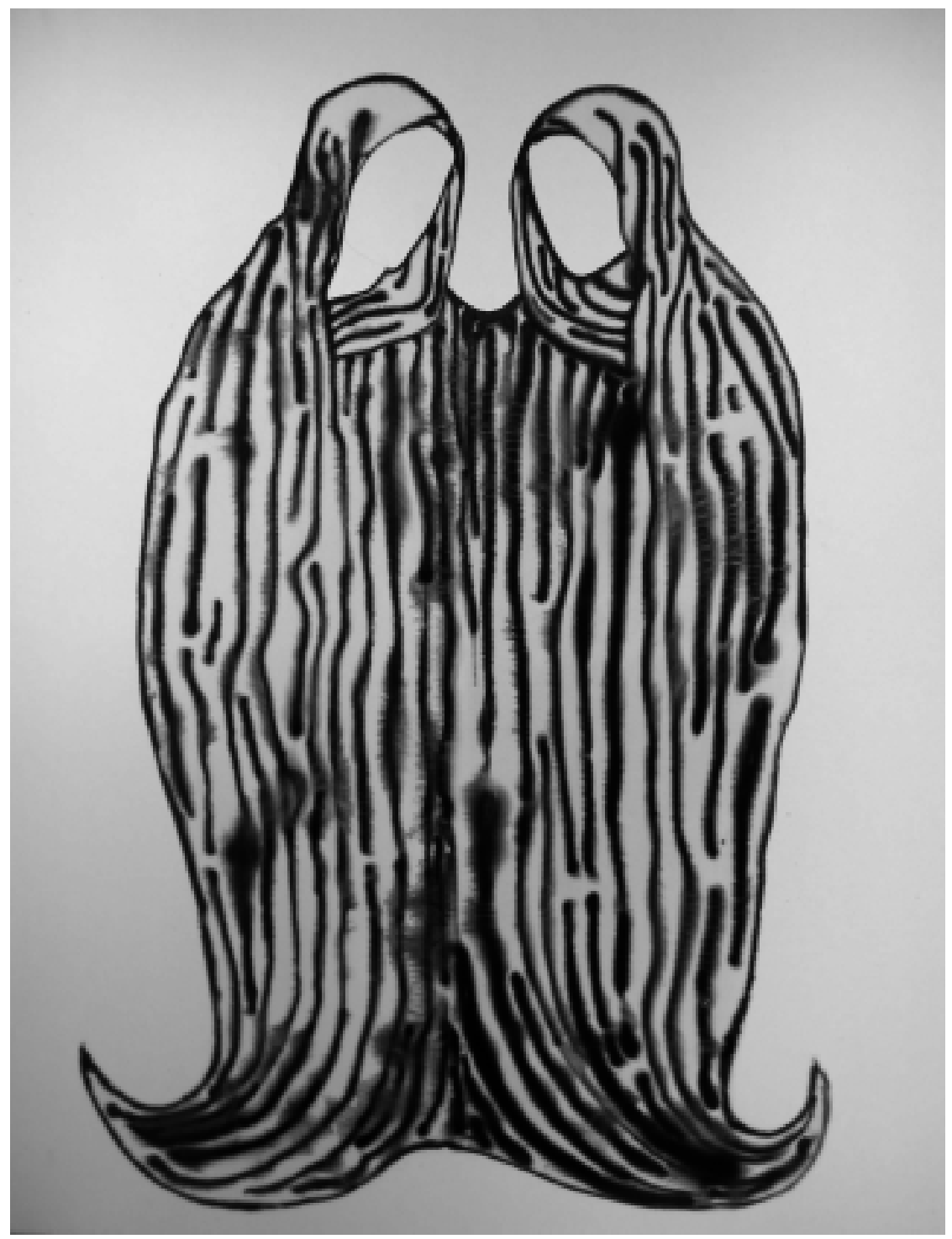

Saintes-Grenouilles, 2007, 213 x $168 \mathrm{~cm}$ (acrylique sur toile). 


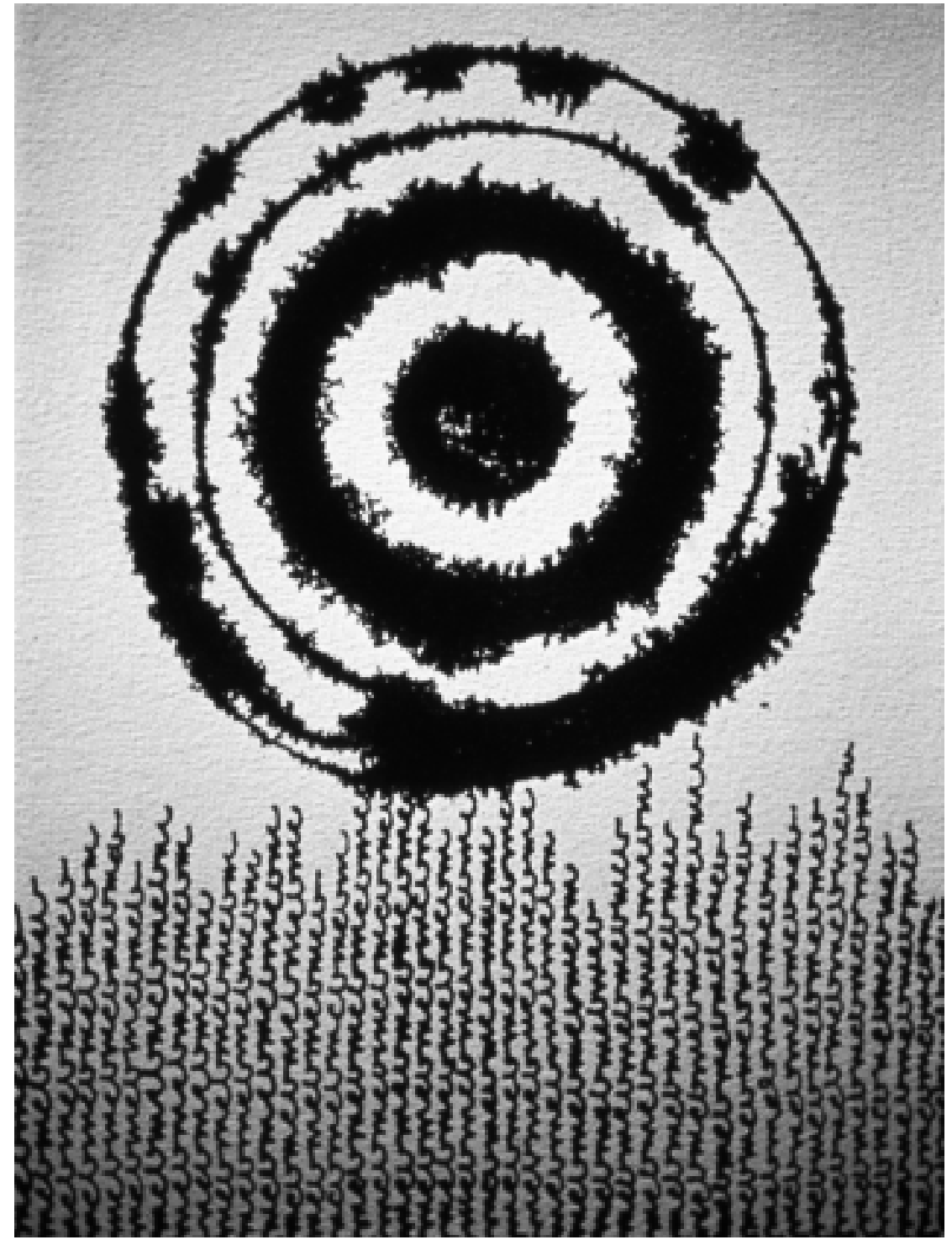

«mcb» petit soleil noir, 2004, $30 \times 45 \mathrm{~cm}$ (encaustique sur toile et encre). 


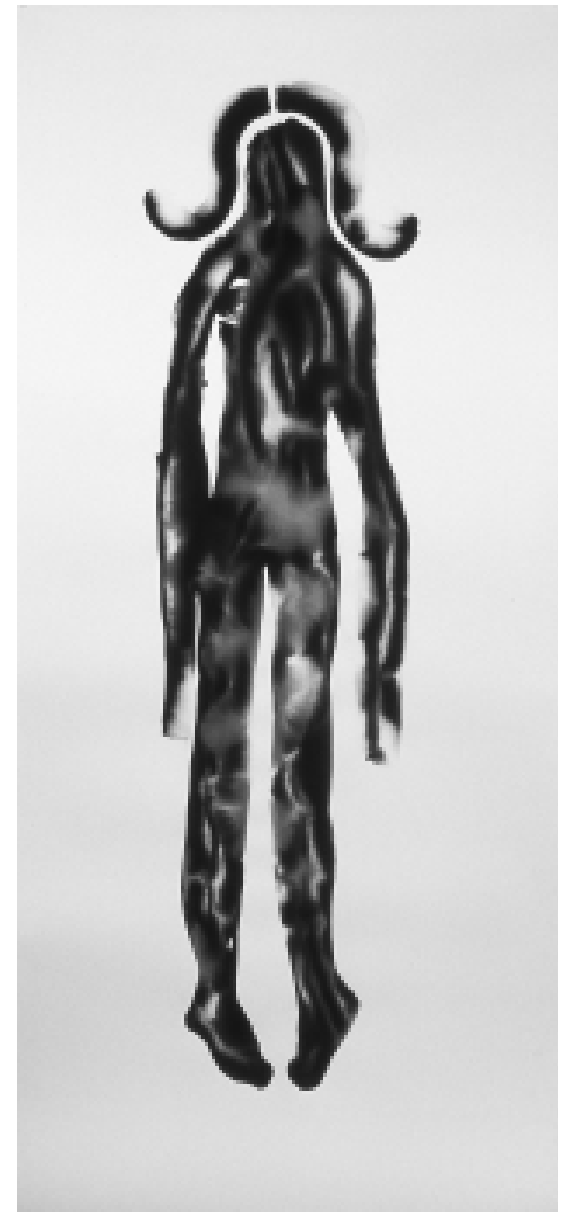

Grand bleu à cornes, 2004, $250 \times 122 \mathrm{~cm}$ (dispersion de pigment sur papier).

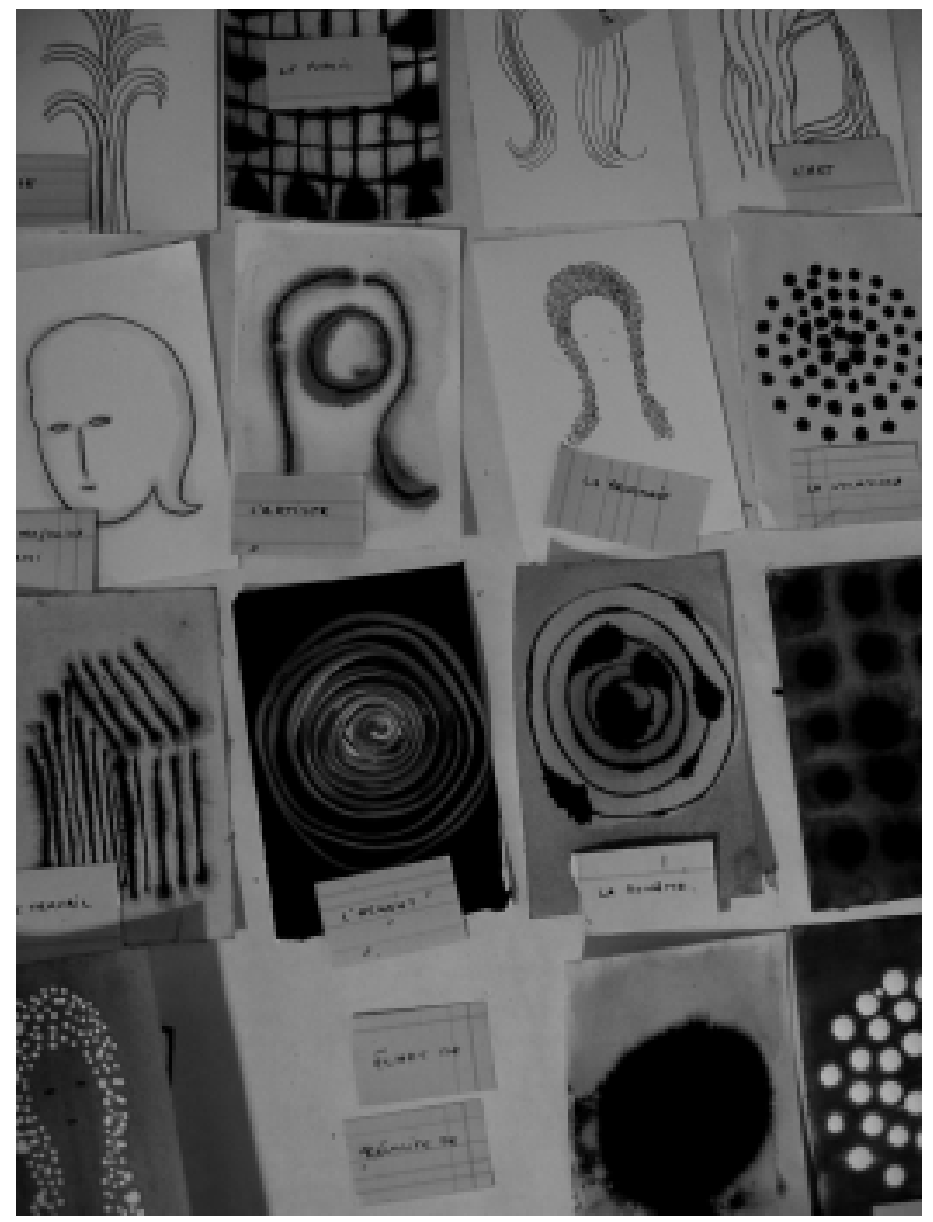

La Vie d'artiste, 2005

formats variés (encres sur papier). 